\title{
Notification that New Names and New Combinations Have Appeared in Volume 46, No. 1, of the IJSB ${ }^{a}$
}

\begin{tabular}{|c|c|c|c|}
\hline Name: & Proposed as: & Authors: & IJSB reference description: \\
\hline Frankiaceae & emend. & Normand et al. & $46(1): 8$ \\
\hline Geodermatophilaceae & emend. & Normand et al. & $46(1): 8$ \\
\hline Devosia & gen. nov. & Nakagawa et al. & 46(1):20 \\
\hline Devosia riboflavina & sp. nov. & Nakagawa et al. & 46(1):20 \\
\hline Rhodococcus percolatus & sp. nov. & Briglia et al. & 46(1):29 \\
\hline Eubacterium minutum & sp. nov. & Poco et al. & 46(1):33 \\
\hline Buttiauxella agrestis & emend. & Müller et al. & 46(1):60 \\
\hline Buttiauxella ferragutiae & sp. nov. & Müller et al. & $46(1): 60$ \\
\hline Buttiauxella gaviniae & sp. nov. & Müller et al. & $46(1): 62$ \\
\hline Buttiauxella brennerae & sp. nov. & Müller et al. & $46(1): 62$ \\
\hline Buttiauxella izardii & sp. nov. & Müller et al. & $46(1): 62$ \\
\hline Buttiauxella noackiae & sp. nov. & Müller et al. & $46(1): 62$ \\
\hline Buttiauxella warmboldiae & sp. nov. & Müller et al. & $46(1): 62$ \\
\hline Kluyvera cochleae & sp. nov. & Müller et al. & 46(1):63 \\
\hline Kluyvera georgiana & sp. nov. & Müller et al. & $46(1): 63$ \\
\hline Bacillus ehimensis & sp. nov. & Kuroshima et al. & $46(1): 79$ \\
\hline Bacillus chitinolyticus & sp. nov. & Kuroshima et al. & 46(1):79 \\
\hline Brachybacterium alimentarium & sp. nov. & Schubert et al. & $46(1): 86$ \\
\hline Brachybacterium tyrofermentans & sp. nov. & Schubert et al. & 46(1):86 \\
\hline Agromyces mediolanus (basonym "Corynebacterium mediolanum") & comb. nov., nom. rev. & Suzuki et al. & 46(1):92 \\
\hline Bacillus haloalkaliphilus & sp. nov. & Fritze & $46(1): 100$ \\
\hline Bacillus carboniphilus & sp. nov. & Fujita et al. & $46(1): 118$ \\
\hline Thermoanaerobacter wiegelii & sp. nov. & Cook et al. & $46(1): 126$ \\
\hline Flavobacterium & emend. & Bernardet et al. & 46(1):139 \\
\hline Flavobacterium aquatile & emend. & Bernardet et al. & 46(1):140 \\
\hline Flavobacterium branchiophilum & emend. & Bernardet et al. & 46(1):140 \\
\hline Flavobacterium columnare (basonym Flexibacter columnaris) & comb. nov. & Bernardet et al. & 46(1):140 \\
\hline Flavobacterium flevense (basonym Cytophaga flevensis) & comb. nov. & Bernardet et al. & $46(1): 141$ \\
\hline Flavobacterium hydatis (basonym Cytophaga aquatilis) & comb. nov., nom. nov. & Bernardet et al. & $46(1): 141$ \\
\hline Flavobacterium johnsoniae (basonym Cytophaga johnsonae) & comb. nov. & Bernardet et al. & $46(1): 141$ \\
\hline Flavobacterium pectinovorum (basonym Cytophaga pectinovora) & comb. nov. & Bernardet et al. & 46(1):141 \\
\hline Flavobacterium psychrophilum (basonym Flexibacter psychrophilus) & comb. nov. & Bernardet et al. & 46(1):142 \\
\hline Flavobacterium saccharophilum (basonym Cytophaga saccharophila) & comb. nov. & Bernardet et al. & 46(1):142 \\
\hline Flavobacterium succinicans (basonym Cytophaga succinicans) & comb. nov. & Bernardet et al. & 46(1):142 \\
\hline Flavobacteriaceae & emend. & Bernardet et al. & 46(1):145 \\
\hline Vibrio ichthyoenteri & sp. nov. & Ishimura et al. & 46(1):159 \\
\hline Helicobacter bizzozeronii & sp. nov. & Hanninen et al. & 46(1): 165 \\
\hline Tolumonas & gen. nov. & Fischer-Romero et al. & $46(1): 187$ \\
\hline Tolumonas auensis & sp. nov. & Fischer-Romero et al. & 46(1):187 \\
\hline Pseudomonas balearica & sp. nov. & Bennasar et al. & 46(1):204 \\
\hline Serpulina pilosicoli & sp. nov. & Trott et al. & 46(1):213 \\
\hline Alteromonas nigrifaciens & emend." & Ivanova et al. & 46(1):227 \\
\hline Spiroplasma diminutum & sp. nov. & Williamson et al. & 46(1):232 \\
\hline Agrococcus & gen. nov. & Groth et al. & 46(1):239 \\
\hline Agrococcus jenensis & sp. nov. & Groth et al. & 46(1):239 \\
\hline Sutterella & gen. nov. & Wexler et al. & 46(1):257 \\
\hline Sutterella wadsworthensis & sp. nov. & Wexler et al. & $46(1): 257$ \\
\hline Nocardia pseudobrasiliensis & sp. nov. & Ruimy et al. & $46(1): 263$ \\
\hline Fervidobacterium gondwanense & sp. nov. & Andrews and Patel & 46(1):268 \\
\hline Paenibacillus larvae & emend. & Heyndrickx et al. & 46(1):277 \\
\hline Paenibacillus larvae subsp. larvae & Rule $46^{c}$ & & \\
\hline Paenibacillus lavae subsp. pulvifaciens (basonym Paenibacillus pulvifaciens) & comb. nov. & Heyndrickx et al. & 46(1):279 \\
\hline Spirochaeta alkalica & sp. nov. & Zhilina et al. & 46(1):309 \\
\hline Spirochaeta africana & sp. nov. & Zhilina et al. & 46(1):310 \\
\hline Spirochaeta asiatica & sp. nov. & Zhilina et al. & 46(1):311 \\
\hline Thermosipho & emend. & Ravot et al. & $46(1): 322$ \\
\hline Lactobacillus casei & Replacement of type strain ${ }^{d}$ & Dicks et al. & $46(1): 340$ \\
\hline Lactobacillus paracasei & nom. rejic. ${ }^{c}$ & Dicks et al. & 46(1):340 \\
\hline Lactobacillus zeae & sp. nov., nom. rev. & Dicks et al. & 46(1):340 \\
\hline Halobacterium salinarum corrig. (Halobacterium salinarium [sic]) & nom. corrig. & Ventosa and Oren & $46(1): 347$ \\
\hline
\end{tabular}

${ }^{a}$ This listing of names published in a previous issue of IJSB is provided as a service to bacteriology to assist in the recognition of new names and new descriptions. This procedure was proposed by the Judicial Commission [Minute II (ii), Int. J. Syst. Bacteriol. 41:185, 1991]. The names given herein have priority according to the issue of the IJSB in which they were published.

${ }^{b}$ Recently, Alteromonas nigrifaciens has been reclassified as Pseudoalteromonas nigrifaciens comb. nov. by Gauthier et al. (IJSB 45:760). The emended description is valid for the new combination, too.

c According to Rule 46 of the International Code of Nomenclature of Bacteria, the valid publication of Paenibacillus larvae subsp. pulvifaciens automatically creates another subspecies, Paenibacillus larvae subsp. larvae (White 1906) Ash et al. 1994 emend. Heyndrickx et al. 1996.

${ }^{a}$ Replacement of a type strain is only a matter of the Judicial Commission. Therefore, this notification is provisional and needs to be awarded by the Judicial Commission.

e According to Rule 56a of the International Code of Nomenclature of Bacteria, only the Judicial Commission can place a name on the list of rejected names. Therefore, this notification is provisional and needs to be awarded by the Judicial Commission. 